\title{
An Attempt to Define the Nature of Chemical Diabetes Using a Multidimensional Analysis
}

\author{
G. M. Reaven and R. G. Miller \\ Departments of Medicine and Statistics, Stanford University and Veterans Administration Hospital, Palo Alto, California, USA
}

\begin{abstract}
Summary. The relationship between chemical diabetes and overt diabetes in 145 nonobese adult subjects has been examined. The degree of glucose intolerance, insulin response to oral glucose, and insulin resistance in normal subjects and patients with nonketotic diabetes were first determined. These variables were then analyzed by a computer program which permits direct visualization of the threedimensional shape of the data set. The picture obtained was that of a boomerang with two wings and a fat middle. The patients were then divided into three groups (normal, chemical diabetes, overt diabetes) on the basis of their oral glucose tolerance. Two-dimensional views of the relationship between the metabolic variables in these subjects resembled the initial three-dimensional image, i. e., a central core with two wings going off in opposite directions. The two wings represented patients with chemical diabetes and overt diabetes, respectively. Following this, the patients were reclassified on the basis of all three metabolic variables (plasma glucose response to oral glucose, plasma insulin response to oral glucose, and degree of insulin resistance) by means of a computer classification which employed a cluster analysis technique. This again resulted in the definition of three groups, in which there was a divergence between subjects classified as having chemical diabetes as contrasted to overt diabetes. This apparent separation between subjects with chemical and overt diabetes may explain why patients with chemical diabetes rarely develop overt diabetes.
\end{abstract}

Key words: Chemical diabetes, multidimensional analysis, insulin resistance, insulin secretion, glucose intolerance, diabetes mellitus.
Approximately ten years ago we published a paper which described the relationship between the plasma glucose and insulin responses to an oral glucose load in normal subjects and patients with varying degrees of hyperglycaemia [1]. At that time we pointed out that the relationship had a "horse shoe" appearance. This description was a function of the fact that individuals with the best glucose tolerance had the lowest insulin levels in response to the oral glucose challenge. In people with less good tolerance, higher accompanying insulin concentrations suggested that glucose removal was impaired in these patients; and that greater amounts of insulin were secreted in an effort to maintain glucose homeostasis. In people with the highest levels of blood glucose, hyperglycaemia did not elicit a significant increase in absolute insulin response. Finally, in patients with the most severe hyperglycaemia, absolute insulin concentrations were low, suggesting a primary impairment of insulin secretion.

Although the existence of this relationship has been subsequently confirmed $[2,3]$, its significance remains unclear. On the one hand, these cross-sectional data could be viewed as depicting the natural history of diabetes, and be interpreted as representing a "Starling curve". This would suggest that within an individual increasing the glycaemic stimulus will lead to an increase in the insulin response, but only up to a certain point. Beta-cell exhaustion eventually supervenes, resulting in a progressive decrease of insulin secretion. Alternatively, the relationship described could result from the fact that different patients have different causes for their hyperglycaemia. Thus, some patients may be primarily insulin resistent (with hyperglycaemia and hyperinsulinaemia), while others are primarily insulin deficient (hyperglycaemia and hypoinsulinaemia). Under 
these conditions the "Starling curve" may be more apparent than real, and the relationship between the plasma glucose and insulin responses to an oral glucose load is a function of the metabolic heterogeneity of the diabetic population. Finally, both may be true, and the distinction between these two types may be blurred in many patients.

At the present time it is not possible to choose between these alternative interpretations of the observed relationship, and the ultimate choice awaits the completion of a proper prospective study. However, in an effort to pursue this question further, we have tried to quantify various aspects of carbohydrate metabolism in nonobese patients with glucose intolerance of varying degrees. We have attempted to gain some insight into the relationship that exists between patients currently being diagnosed as diabetic on the basis of three metabolic variables: glycaemic response to a glucose challenge, insulin response to a glucose challenge, and insulin sensitivity.

\section{Methods}

\section{Experimental Protocol}

The data to the presented have been derived from investigation of 145 nonobese individuals who volunteered to take part in our announced studies of the etiology of diabetes. Their stated reasons for participation were varied, and include a recent diagnosis of diabetes, a family history of diabetes, a desire to have a thorough physical examination, or a wish to be part of a research program. After giving informed consent all subjects were admitted to the Stanford Clinical Research Center. None of the subjects were receiving insulin or oral hypoglycaemic agents. They were not taking any medications known to modify carbohydrate metabolism, and they had no clinical or laboratory evidence of hepatic or cardiovascular disease. Their relative weights varied between 0.85 and 1.15 . They consumed a liquid weight-maintenance formula diet, consisting of $43 \%$ carbohydrate, $42 \%$ fat and $15 \%$ protein, three times daily in portions of one-fifth, two-fifths, and two-fifths of total calories. Daily weights were obtained and weight changes during the study period did not exceed $1 \mathrm{~kg}$.

After at least three days of this diet, subjects were given an oral glucose load of $40 \mathrm{~g} / \mathrm{m}^{2}$ body surface area, and blood samples were drawn during the following three hours for measurement of plasma glucose and immunoreactive insulin levels.

Two days later, after a 14 hour overnight fast, all subjects received an intravenous injection of $5 \mathrm{mg}$ of propranolol, followed in five $\mathrm{min}$ by a constant intravenous infusion of glucose $(6 \mathrm{mg} / \mathrm{kg} /$ $\mathrm{min})$, insulin $(80 \mathrm{mU} / \mathrm{min})$, epinephrine $(6 \mu \mathrm{g} / \mathrm{min})$ and propranolol $(0.08 \mathrm{mg} / \mathrm{min})$. The solution was infused continuously for $150 \mathrm{~min}$ into an antecubital vein. Blood samples were drawn from the opposite arm through a needle kept patent by a slow saline $(0.154 \mathrm{~mol} / \mathrm{l})$ infusion every $5 \mathrm{~min}$ for 120 to $150 \mathrm{~min}$. Under these experimental conditions, endogenous insulin secretion is suppressed and steady state plasma glucose (SSPG) and insulin (SSPI) levels are reached by $90 \mathrm{~min}$ [4]. Studies in which the coefficients of variation of the individual SSPG and SSPI values were greater than $10 \%$ were excluded from analysis. Since similar SSPI values are achieved in all subjects, this technique allows us to compare the ability of different subjects to dispose of identical glucose loads under the same insulin stimulus. Therefore, the SSPG values are a measure of efficiency of insulin-mediated glucose utilization, i. e., insulin resistance.

\section{Chemical Analysis}

Blood for determination of plasma glucose and insulin was drawn into test tubes containing EDTA, the plasma quickly separated, and aliquots stored at $-20^{\circ} \mathrm{C}$ until subsequent analysis for insulin [5], and glucose using a Glucose Analyzer (Beckman Instruments, Fullerton, Cal).

\section{Data Analysis}

For each subject six variables were measured and entered onto punch cards for computer analysis. The variables used were age, relative weight, fasting plasma glucose, area under the plasma glucose curve for the three hour oral glucose tolerance test (OGTT), area under the plasma insulin curve for the OGTT, and the steady state plasma glucose response (SSPG). The OGTT was only carried out for three hours, and plasma glucose and insulin values did not return to baseline in all patients during this interval. If these tests had been carried out longer, it would certainly have changed the quantitative nature of the results.

The data for the 145 subjects were inspected with the aid of the Prim 9 program at the Stanford Linear Accelerator Computation Center. This program allows one to see any two-dimensional projection of a three-dimensional data set, and through continuous rotation of the axes the viewer can discern the three-dimensional shape of the data set. Different sets of three variables out of the six were examined, but attention mainly focused on glucose area, insulin area, and SSPG for the following reasons.

Two-dimensional scattergrams of all possible combinations were constructed. The plots of age and relative weight versus the other variables exhibited little or no association. These variables were therefore dropped from subsequent analysis. On the other hand, fasting plasma glucose exhibited a very high degree of linear association with glucose area $(r=0.96)$. Since the two variables, fasting plasma glucose and glucose area, were essentially equivalent, there seemed little point in using both and fasting blood glucose was omitted. The subsequent analysis, therefore, only considered the relationship between plasma glucose response, plasma insulin response, and SSPG response.

\section{Results}

Using the Prim 9 program, the image that the viewers had of the set of 145 three-dimensional data points was that of a boomerang with floppy wings and a fat middle (Fig. 1). Given this visual perception of the three-dimensional relationship between the various metabolic variables chosen for examination, it seemed unlikely that the 145 subjects studied belonged to one population. It is difficult to conceptualize this three-dimensional perception in two dimensions. In an attempt to do so, we have drawn three scattergrams which illustrate all of the possible two-dimensional relationships between the three metabolic variables. In these figures we have divided the experimental subjects into three groups using conventional clinical criteria. One group was defined as having fasting hyperglycaemia (plasma glucose levels in excess of $110 \mathrm{mg} / \mathrm{dl}$ ), and these patients were considered to have overt diabetes. The remaining subjects were subdivided into two groups: (1) those with plasma glucose levels greater than $185 \mathrm{mg} / \mathrm{dl}$ and $140 \mathrm{mg} / \mathrm{dl}$ at one and two hours after 


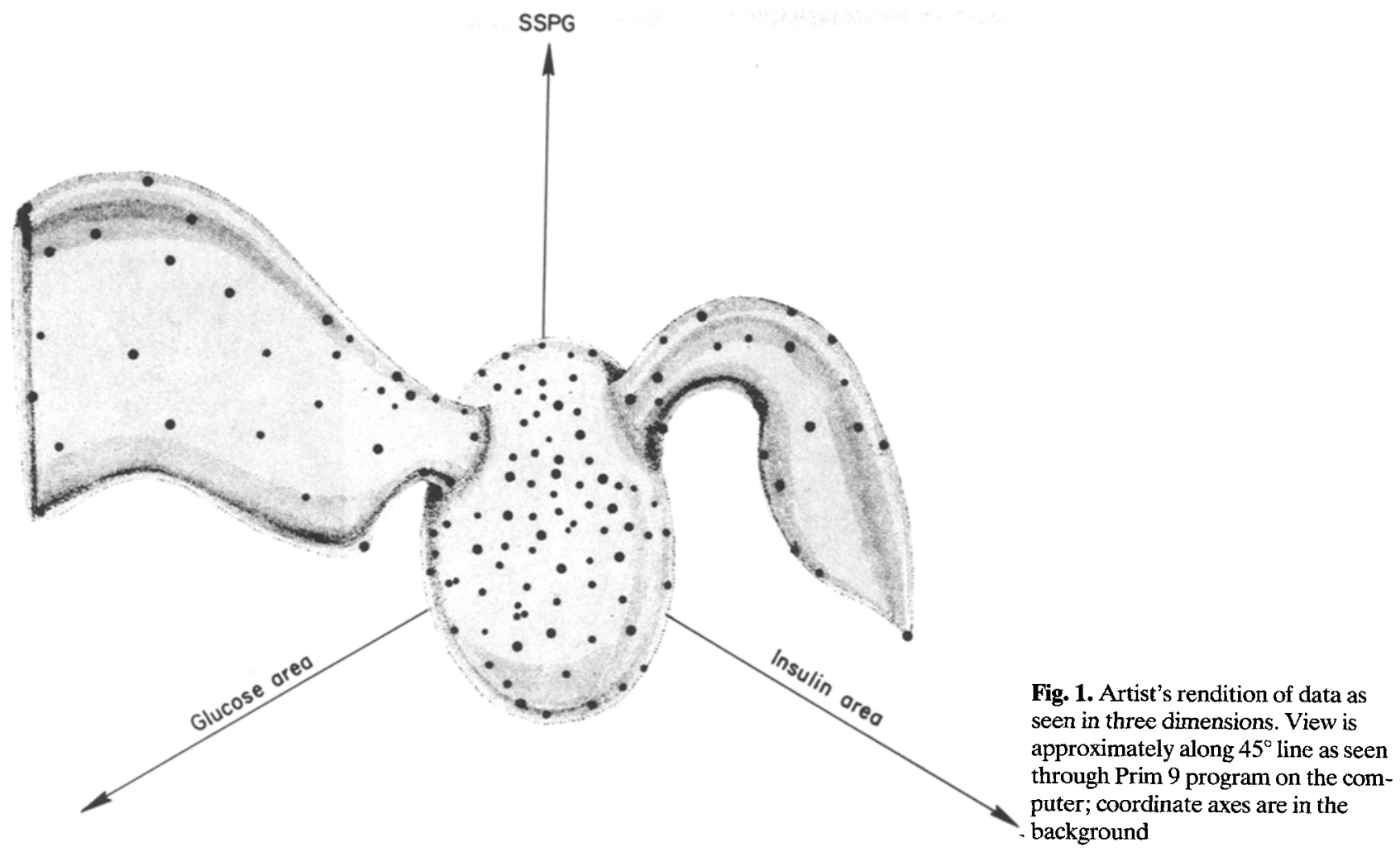

Table 1. Classification of the 145 subjects into three groups on the basis of the oral glucose tolerance test

\begin{tabular}{llllll}
\hline \multirow{2}{*}{ Group } & Number & Retabolic characteristics (mean \pm SD) & & \\
\cline { 3 - 6 } & & Rel.wt. & $\begin{array}{l}\text { Glucose area } \\
(\mathrm{mg} / 100 \mathrm{ml} \cdot \mathrm{hr})\end{array}$ & $\begin{array}{l}\text { Insulin area } \\
(\mu \mathrm{U} / \mathrm{ml} \cdot \mathrm{hr})\end{array}$ & $\begin{array}{l}\text { SSPG } \\
(\mathrm{mg} / 100 \mathrm{ml})\end{array}$ \\
\hline Normal & & & $350 \pm 37$ & $173 \pm 69$ & $114 \pm 58$ \\
Chemical diabetes & 76 & $0.98 \pm 0.11$ & $494 \pm 55$ & $288 \pm 158$ & $209 \pm 60$ \\
Overt diabetes & 36 & $1.02 \pm 0.10$ & $1044 \pm 309$ & $106 \pm 93$ & $319 \pm 88$ \\
\hline
\end{tabular}

the glucose load (chemical diabetes) and (2) a normal group. Using the basis of these criteria the patient population consisted of 76 normal subjects, 36 individuals with chemical diabetes, and 33 patients with diabetes. The mean $( \pm S E M)$ values of the three patient groups for relative weight, glucose and insulin responses during the OGTT, and SSPG are seen in Table 1.

Figure 2 depicts the relationship between the plasma glucose and insulin responses in the three clinical groups. The patients with chemical diabetes seem to be going off in a different direction (up) as compared to subjects with fasting hyperglycaemia (to the right). Figure 3 displays the relationship between insulin response and insulin resistance (SSPG). As in Figure 2, patients with chemical diabetes and fasting hyperglycaemia cluster in different areas. Figure 4 depicts the relationship between glucose response and SSPG. This two-dimensional representation of the three-dimensional relationship is different than that seen in Figs. 2 and 3, and is the only view of the data consistent with the existence of one diabetic patient population.

It is obvious that the patient population has been divided into three groups on the basis of diagnostic decisions which took into account only one aspect of the carbohydrate metabolism of these individuals. In order to provide a more comprehensive description of each patient, the plasma insulin response during the OGTT and the estimate of insulin resistance (SSPG) were included with the plasma glucose response to achieve a new data set which now included three metabolic variables. Furthermore, in an effort to avoid the rigidity of the arbitrary clinical classification that formed the basis of Figs. 2-4, we employed the cluster analysis technique described by Friedman and Rubin [6]. This enabled us to develop a computer classification of each patient which took into account all three metabolic variables and was independent of a priori clinical judgements. Initial 

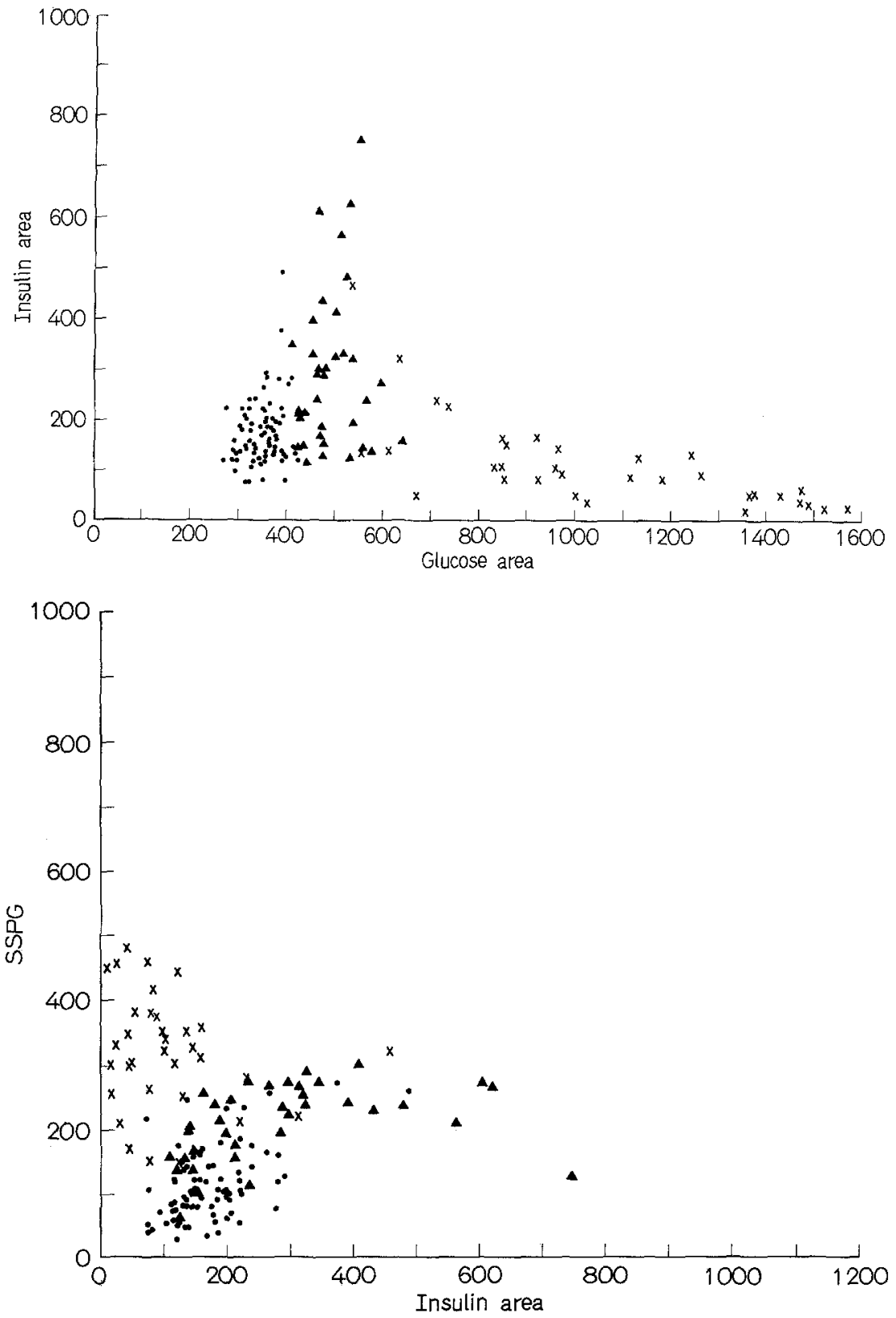

Fig. 2. Relationship between the plasma glucose $(\mathrm{mg} / 100 \mathrm{ml} \cdot \mathrm{hr})$ and insulin $(\mu \mathrm{U} / \mathrm{ml} \cdot \mathrm{hr})$ responses during the OGTT for all 145 subjects. Subjects have been defined as being normal $(\boldsymbol{\bullet})$, or as having either chemical $(\mathbf{A})$ or overt $(\mathrm{X})$ diabetes on the basis of the results of the OGTT starting values for the three variables (plasma glucose and insulin response during the OGTT and SSPG response) were created for each group from average values of the same three variables found in an earlier study [7]. At the first pass the computer then assigned each subject to one of the three groups, depending on which initial group values were closest to his/her values in terms of Euclidean distance. After all 145 subjects had been assigned to one of the three groups, means of the values for the subjects in each group were computed. These means were then used as the typical group values at the second computer pass, and each subject was reassigned to the group whose mean values were closest to his/her values. Again, group means were computed, and this process was continued until convergence occurred, i. e., until no subject was reassigned to a different group at the next pass.

The results of this procedure are seen in Table 2, in which are summarized the new diagnostic assignments, the mean values of the three metabolic variables for each of the diagnostic categories, as well as the values which were used as the initial estimates. It is clear from comparison of Tables 1 and 2 that the use of the clustering approach led to a classification of the patient population which resembled the original one in terms of both numbers of patients within each category and mean values for the three metabolic variables measured. However, there were 20 instances in which patients were classified differ- 
Table 2. Computer classification of the 145 subjects into three groups. Initial starting values for the clustering algorithm are displayed in parentheses

\begin{tabular}{|c|c|c|c|c|}
\hline \multirow[b]{2}{*}{ Group } & \multirow[b]{2}{*}{ Number } & \multicolumn{3}{|c|}{ Metabolic characteristics (mean $\pm S D$ ) } \\
\hline & & $\begin{array}{l}\text { Glucose area } \\
(\mathrm{mg} / 100 \mathrm{ml} \cdot \mathrm{hr})\end{array}$ & $\begin{array}{l}\text { Insulin area } \\
(\mu \mathrm{U} / \mathrm{ml} \cdot \mathrm{hr})\end{array}$ & $\begin{array}{l}\text { SSPG } \\
(\mathrm{mg} / 100 \mathrm{ml})\end{array}$ \\
\hline Normal & 84 & $\underset{(335)}{363 \pm 54}$ & $\begin{array}{l}164 \pm 51 \\
(164)\end{array}$ & $\begin{array}{l}113 \pm 51 \\
(120)\end{array}$ \\
\hline Chemical diabetes & 35 & $\begin{array}{l}524 \pm 86 \\
(505)\end{array}$ & $321 \pm 157$ & $\begin{array}{l}236 \pm 47 \\
(225)\end{array}$ \\
\hline Overt diabetes & 26 & $\begin{array}{l}1153 \pm 251 \\
(893)\end{array}$ & $\begin{array}{l}76 \pm 46 \\
(108)\end{array}$ & $\begin{array}{l}343 \pm 79 \\
(325)\end{array}$ \\
\hline
\end{tabular}
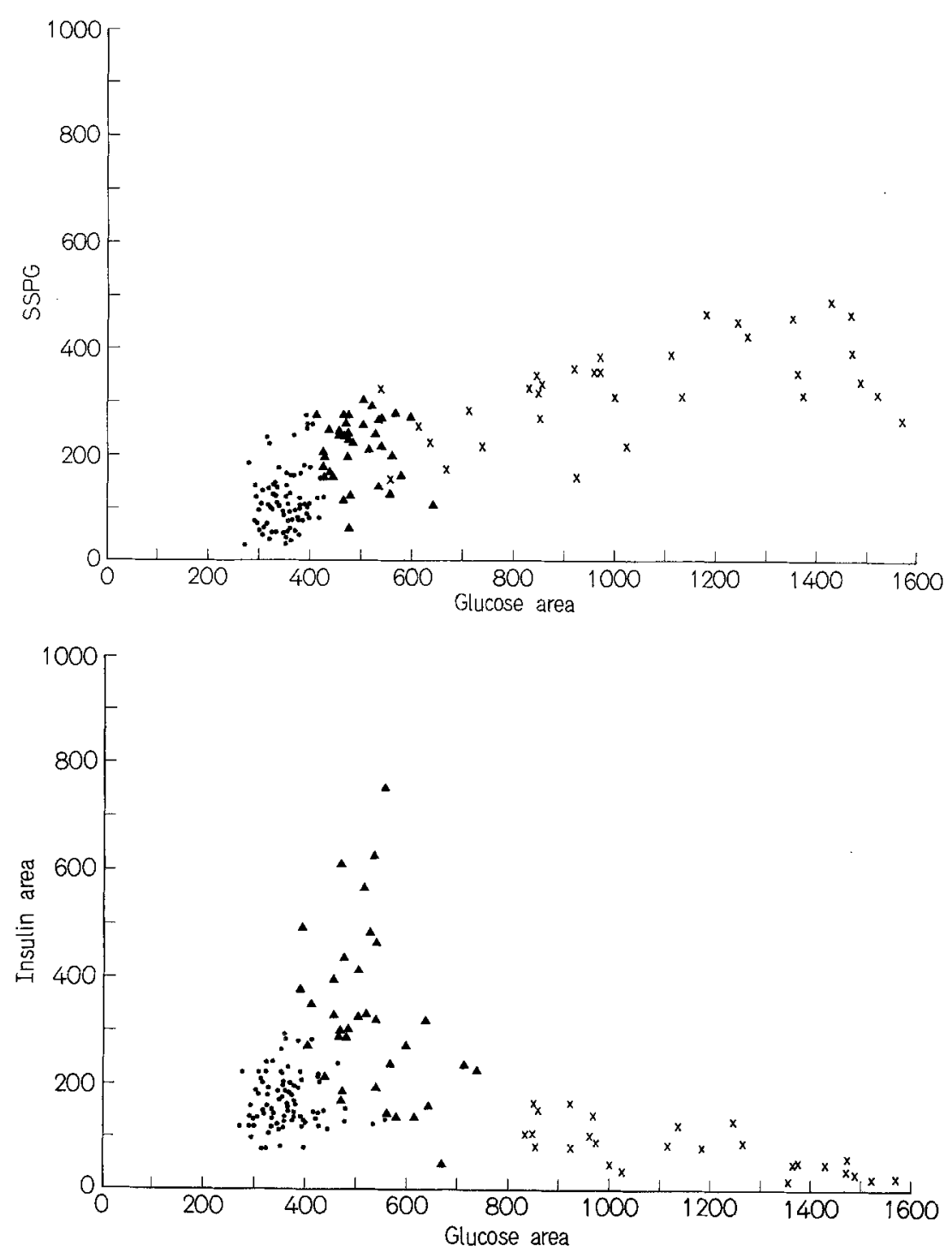

Fig. 4. Relationship between the plasma glucose response $(\mathrm{mg} / 100 \mathrm{ml} \cdot \mathrm{hr})$ during the OGTT and SSPG $(\mathrm{mg} / 100 \mathrm{ml})$ for all 145 subjects. Subjects have been defined as being normal $(\bullet)$, or as having either chemical $(\boldsymbol{A})$ or overt $(\mathrm{X})$ diabetes on the basis of the results of the OGTT ently by these two approaches. Ten patients with the original diagnosis of chemical diabetes were computer classified as normal. All were borderline on the original diagnosis. Six patients with lesser degrees of fasting hyperglycaemia $(110-140 \mathrm{mg} / \mathrm{dl})$ were reclas- sified as having chemical diabetes. Three patients were moved from the normal to the chemical diabetes group. All three of these subjects were hyperinsulinemic and insulin resistant, but managed to maintain normal glucose tolerance. The last instance of 


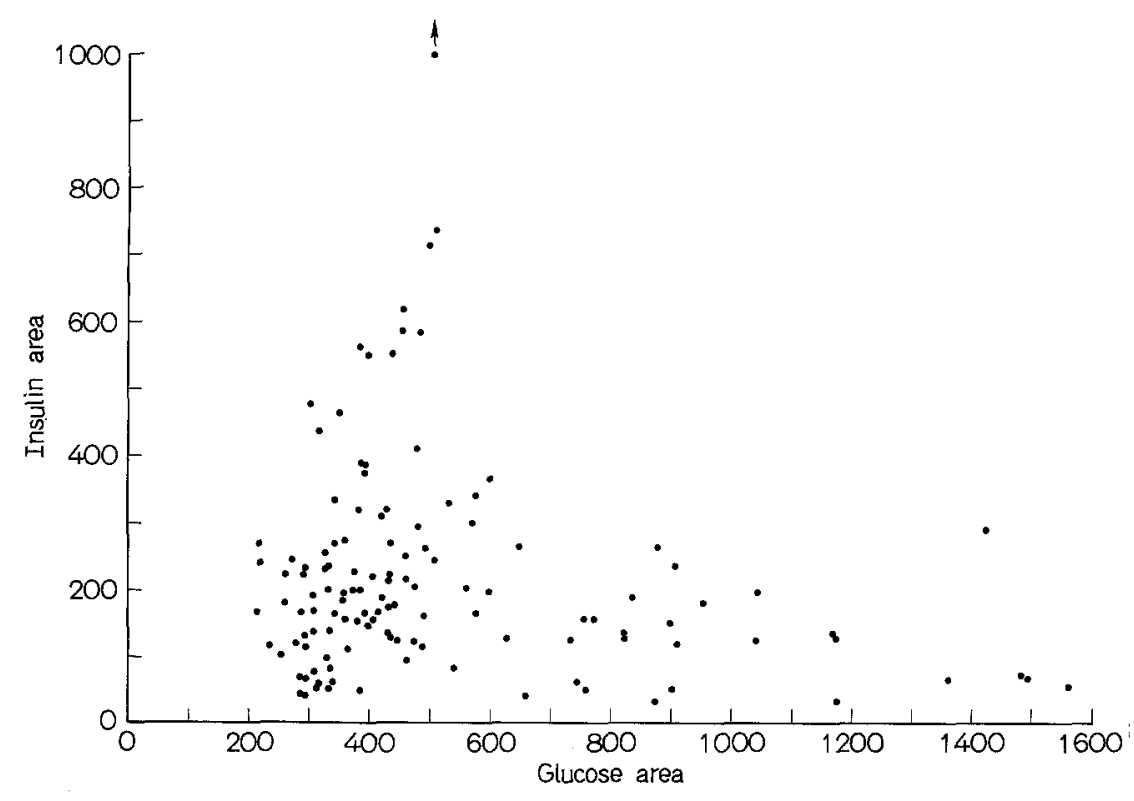

Fig. 6. Relationship between the plasma glucose $(\mathrm{mg} / 100 \mathrm{ml} \cdot \mathrm{hr})$ and insulin $(\mu \mathrm{U} / \mathrm{ml} \cdot \mathrm{hr})$ responses during an OGTT for 125 subjects studied by Reaven and Miller in 1968 [5] reclassification involved a patient with mild fasting hyperglycaemia, whose plasma glucose, insulin and SSPG responses were all very close to those of normal subjects. In all instances the reclassification process focused on group similarities and differences, and as such offers as alternative approach to the diagnosis of various kinds of diabetes. At the least, cluster analysis enables us to define patient categories in which all of those included within a given group share several metabolic characteristics.

Two-dimensional scattergrams displaying the relationship between the three metabolic variables for the computer-derived patient groups were also constructed. Figure 5 describes the relationship between plasma glucose and insulin responses during the OGTT. The data displayed in this scattergrams appears to represent two wings emanating from a central core, and the resemblance to both the artist's depiction of the three-dimensional data set (Fig. 1) and to Figure 2 is apparent. However, the apparent separation between patients with chemical diabetes from those with significant fasting hyperglycaemia is even more striking in Figure 5. Similarly, two-dimensional scattergrams (not shown for the sake of brevity) which described the relationship between insulin response and insulin resistance, and between glucose response and insulin resistance, closely resembled Figs. 3 and 4, respectively. However, in both instances the cohesiveness of the three patient groups was increased in a fashion similar to the comparison between Figures 2 and 5.

\section{Discussion}

These studies were undertaken in order to gain insight into the significance of the relationship between the plasma glucose and insulin responses to oral glucose in nonketotic diabetes. More specifically, it represents an attempt to determine if the cross-sectional relationship that has been observed between these two variables depicts the natural history of diabetes, or simply reflects the metabolic heterogeneity of patients currently classified as having diabetes. In this context, the significance of the patient with chemical diabetes looms large. Can we continue to view these patients with the smallest detectable abnormality of glucose tolerance as representing the beginning of a syndrome, which at the other end of the spectrum consists of patients with insulin-dependent diabetes [8]? This question is particularly relevant in view of the observation that relatively few patients with the mildest form of glucose intolerance seem to show much in the way of progressive deterioration of glucose tolerance with time [8-10]. A possible explanation for this lack of progression is recent evidence attesting to the heterogeneity of patients currently classified as having diabetes. For example, it has recently been suggested that idiopathic diabetes mellitus can be divided into at least two general disease processes on the basis of differences in autoimmunity [11]. This subdivisions of diabetes into two general classes on the basis of autoimmunity appears to be supported by genetic studies, in which different patterns of inheritance seem to be present in young patients who have insulin-dependent diabetes as compared to similarly aged individuals with insulin-independent diabetes [12]. Finally, patients currently classified as having diabetes seem to break down into two general categories on the basis of pancreatic islet cell function - those who retain the ability to secrete substantial amounts of insulin and those who are insulin deficient [1-3].

Metabolic heterogeneity also exists in patients 
with chemical diabetes, and glucose intolerance in this situation may be associated with either insulin deficiency or insulin resistance $[8,13]$. Furthermore, it has been suggested that the patients with chemical diabetes who progress to insulin requiring diabetes may be the ones in whom insulin deficiency was the initial lesion [13-15]. A possible corollary to this observation is the fact [17] that 13 of $20(65 \%)$ of recently diagnosed patients with relatively mild diabetes at the onset, and positive pancreatic islet cell antibodies, progressed to insulin-requiring diabetes. In contrast, only 14 of $159(9 \%)$ of similar patients, without islet cell antibodies, progressed in a similar fashion during the period of observation.

These observation are particularly relevant in light of a recent publication from our laboratory [13], in which we measured both insulin response and insulin resistance in patients with chemical diabetes. There was a statistically significant positive relationship between these two variables in both normal subjects and patients with impaired glucose tolerance, and most patients with chemical diabetes had both hyperinsulinaemia and increased insulin resistance. Whether the hyperinsulinaemia is the cause or the result of the insulin resistance is unclear, but in either event it seems difficult to imagine that the majority of patients with insulin deficiency and overt diabetes evolved from a population of patients with these metabolic characteristics.

We feel that the results we have presented lend further credence to this point of view for several reasons. In the first place, we believe that the actual experimental data are representative of the world at large. This conclusion is based upon the fact that there is a close similarity between the present observations on 145 subjects (see Figs. 2 and 5) and those we described [1] on 125 patients 10 years ago (Fig. 6). Secondly, we have attempted to pursue this inquiry by focusing upon more than one facet of patients with diabetes. In order to accomplish this we have chosen to examine three different aspects of carbohydrate metabolism - degree of glucose intolerance, insulin response to a glucose challenge, and the efficiency with which insulin disposes of a glucose load (insulin sensitivity). Other possibilities such as fasting glucose, relative body weight and age were shown to contribute little additional useful information. It is obvious that selection of other metabolic characteristics could have led to a different interpretation, but it is equally obvious that the variables chosen to characterize the patients - glucose response, insulin response and insulin sensitivity are relevant ones. Thirdly, we have used a variety of analytical approaches in an effort to define the relationship between the experimental population. When the variables chosen were subjected to a threedimensional analysis (Prim 9 program), we did not see one population (Fig. 1). Furthermore, twodimensional scattergrams (Figs. 2, 3 and 4) based upon a clinical classification of the patients which only utilized degree of glucose intolerance, seemed to support this view. This was also true (see Fig. 5) when patients were subjected to cluster analysis and the three metabolic variables used to generate a classification of patients which was not dependent upon arbitrary clinical decisions based upon degree of glucose intolerance. Irrespective of the mode of data analysis, patients with chemical diabetes seemed to be quite disparate from those with overt diabetes, and it is difficult to imagine that patients with overt diabetes by and large evolve from patients with chemical diabetes. Although the relationship between patients with overt and chemical diabetes seems to suggest the relationship of two unrelated groups, it is difficult from the current analysis to tell whether normals and chemical diabetics constitute one group with a long tail or two groups. Perhaps the simplest conclusion from the current data would be to consider that both overt diabetics and chemical diabetics can be derived from the normal population.

The only observation in the current study which is in conflict with the view that patients with chemical and overt diabetes do not make up one population is the relationship depicted in Fig. 4 between degree of glucose intolerance (glucose area), and severity of insulin resistance (SSPG). This two-dimensional representation of the three-dimensional relationship is compatible with the notion that the three groups make up a continuous spectrum. This is undoubtedly a function of the fact that insulin resistance is a common finding in patients with nonketotic diabetes, and the greater the degree of glucose intolerance, the more severe the insulin resistance $[17,18]$. However, it is likely that the insulin resistance is a primary defect in patients with chemical diabetes, whereas it appears to be a secondary manifestation in subjects with severe insulin deficiency $[7,19,20]$. Thus, the apparent relationship between glucose intolerance and insulin resistance in these patients appears to be a function of different metabolic events resulting from the heterogeneity of patients currently classified as having diabetes.

Finally, it is obvious that the above conclusions are based upon generalizations concerning the metabolic characteristics of patients with chemical diabetes - specifically, that their mild glucose intolerance is associated with a loss of insulin sensitivity, without a primary defect in the insulin secretory response to oral glucose. It is apparent that not all patients with chemical diabetes share these metabolic characteristics $[8,13-15]$ and that the glucose intolerance of some proportion of patients with chemical diabetes is associated with a defect in the plasma insulin response to glucose. As such, these patients 
may well represent the individuals who progress to overt diabetes $[8,10,13-15]$. On the other hand, the data presented suggest that the metabolic characteristics of the patients studied are such as to make it unlikely that the majority of subjects currently classified as having chemical diabetes evolve into patients with overt diabetes. Furthermore, these differences between the metabolic characteristics of patients currently classified as having diabetes may well represent the metabolic corollary of the differences in inheritance and immune response that are now known to exist in such patients.

Acknowledgements. We wish to thank M. Fisherkeller, J. Halpern, B. Eynon, M. Jedd and J.Moore for their excellent technical assistance. This work was supported in part by grants from the National Institutes of Health, NHLI \#HL 08506, NIGMS \#GM 21215, and \#RR-70 from the General Clinical Research Centers Branch, and from the Research Services of the Veterans Administration.

\section{References}

1. Reaven, G. M., Miller, R.: Study of the relationship between glucose and insulin responses to an oral glucose load. Diabetes 17, 560-569 (1968)

2. Chiles, R., Tzagoumis, M.: Excessive serum insulin response to oral glucose in obesity and mild diabetes. Diabetes 19 , 458-464 (1970)

3. Savage, P. J., Dippe, S. E., Bennett, P. H., Gorden, P., Roth, J., Rushforth, N. B., Miller, M.: Hyperinsulinemia and hypoinsulinemia. Diabetes 24, 362-368 (1975)

4. Shen, S.-W., Reaven, G. M., Farquhar, J. W.: Comparison of impedance to insulin mediated glucose uptake in normal and diabetic subjects. J. Clin. Invest. 49, 2151-2160 (1970)

5. Desbuquois, B., Aurbach, G. D.: Use of polyethylene glycol to separate free and antibody-bound peptide hormones in radioimmunoassays. J. Clin. Endocrinol. Metab. 33, 732-738 (1971)

6. Friedman, H.P., Rubin, J.: On some invariant criteria for grouping data. J. Am. Statist. Assoc. 62, 1159-1178 (1967)

7. Reaven, G. M., Bernstein, R., Davis, B., Olefsky, J. M.: Nonketotic diabetes mellitus: insulin deficiency or insulin resistance? Am. J. Med. 60, 80-88 (1976)

8. Fajans, S. S.: The natural history of idiopathic diabetes mellitus. Heterogeneity of insulin responses in latent diabetes. In: The Genetics of Diabetes Mellitus. Creutzfeldt, W., Köbber- ling, J., Neel, J. V. (Ed.), pp. 64-78. Berlin, Heidelberg, New York: Springer 1976

9. O'Sullivan, J. B., Mahan, C. M.: Prospective study of 352 young patients with chemical diabetes. N. Engl. J. Med. 278, 1038-1041 (1968)

10. Rosenbloom, A. L., Drash, A., Guthrie, R.: Conference summary: chemical diabetes in childhood. Metabolism 22, 413-419 (1973)

11. Irvine, W. J.: Classification of idiopathic diabetes. Lancet 1977 II, 638-642

12. Tattersall, R. B., Fajans, S. S.: A difference between the inheritance of classical juvenile-onset and maturity-onset type diabetes of young people. Diabetes 24, 44-53 (1975)

13. Reaven, G.M., Olefsky, J.M.: Relationship between heterogeneity of insulin responses and insulin resistance in normal subjects and patients with chemical diabetes. Diabetologia 13, 201-206 (1977)

14. Rosenbloom, A. L., Wheeler, L., Bianchi, R., Chin, F.T., Tiwary, C. M., Grgic, A.: Age-adjusted analysis of insulin responses during normal and abnormal glucose tolerance tests in children and adolescents. Diabetes 24, 820-828 (1975)

15. Fajans, S. S., Floyd, Jr., J. C., Tattersall, R. B., Williamson, J. R., Pek, S., Taylor, C. I.: The various faces of diabetes in the young. Arch. Intern. Med. 136, 194-202 (1976)

16. Irvine, W. J., Gray, R. S., McCallum, C. J., Duncan, L. J. P.: Clinical and pathogenic significance of pancreatic-islet-cell antibodies in diabetics treated with oral hypoglycaemic agents. Lancet 1977 I, 1025-1027

17. Ginsberg, H., Olefsky, J. M., Reaven, G. M.: Further evidence that insulin resistance exists in patients with chemical diabetes. Diabetes 23, 674-678 (1974)

18. Ginsberg, H., Kimmerling, G., Olefsky, J. M., Reaven, G. M.: Demonstration of insulin resistance in untreated adult onset diabetic subjects with fasting hyperglycemia. J. Clin. Invest. 55, 454-461 (1975)

19. Schoenle, E., Zapf, J., Froesch, E. R.: Effects of insulin and NSILA on adipocytes of normal and diabetic rats: receptor binding, glucose transport and glucose metabolism. Diabetologia 13, 243-249 (1977)

20. Reaven, G. M., Sageman, W. S., Swenson, R. S.: Development of insulin resistance in normal dogs following alloxan induced insulin deficiency. Diabetologia 13, 459-462 (1977)

Received: September 13, 1977,

and in revised form: August 10, 1978

Gerald M. Reaven, M. D.

Veterans Administration Hospital (111-M)

3801 Miranda Avenue

Palo Alto, CA 94304

USA 